\title{
A Short Polarization Converter Using a Triangular Waveguide
}

\author{
Junji Yamauchi, Member, IEEE, Member, OSA, Masahiro Yamanoue, and Hisamatsu Nakano, Fellow, IEEE
}

\begin{abstract}
A novel polarization converter using a triangular waveguide is proposed and analyzed by the imaginary-distance beam-propagation method based on Yee's mesh and the finite-difference time-domain method. The polarization conversion length is investigated as a function of relative refractive index difference. It is found, for a silicon core embedded in a silica cladding, that the conversion length is $2 \mu \mathrm{m}$, while the insertion loss is $0.5 \mathrm{~dB}$ at a wavelength of $1.55 \mu \mathrm{m}$. The extinction ratio is more than $20 \mathrm{~dB}$ over a wide wavelength range of 1.25 to $1.65 \mu \mathrm{m}$. Using a geometrically expanded model, the polarization conversion behavior is verified in the experiment at a microwave frequency of $15 \mathrm{GHz}$. Finally, reasonable polarization conversion is obtained with a modified structure, in which the two corners of the triangular waveguide are cut and the cut plane is aligned with a square input (output) waveguide.
\end{abstract}

Index Terms-Beam-propagation method, finite-difference time-domain (FDTD) method, polarization converter, triangular waveguide, Yee's mesh.

\section{INTRODUCTION}

A POLARIZATION converter has many applications [1], including polarization controllers, switches, and polarization diversity heterodyne receivers. After investigating a longitudinally periodic structure [2], converters based on a single asymmetric sloped rib waveguide have been proposed and investigated [3]-[9]. These converters are based on beating of two orthogonal modes, and a high index contrast is required to achieve a short conversion length. Recently, a polarization converter in a buried waveguide has also received attention [10], which is based on adiabatic mode transitions. It should be noted, however, that the previous waveguide-type converters have a device length of more than $70 \mu \mathrm{m}$. An ultrasmall polarization rotator based on an off-axis double-core structure, which was reported most recently, still has a device length of $50 \mu \mathrm{m}$ [11].

In this paper, a novel short polarization converter using a triangular waveguide is proposed [12] and studied. The device length of the present converter is at least one order of magnitude less than that of the previous waveguide-type structures. The polarization conversion behavior is evaluated by the imaginary-distance beam-propagation method based on Yee's mesh (YM-BPM) [13] and the finite-difference time-domain (FDTD) method. It is found for a silicon core embedded in a silica cladding that the polarization conversion length is only $2 \mu \mathrm{m}$, while the insertion loss is less than $0.5 \mathrm{~dB}$, at a wavelength of

Manuscript received October 31, 2007; revised January 18, 2008. This work was supported by MEXT Grant-in-Aid for Scientific Research (C) (19560355).

The authors are with Faculty of Engineering, Hosei University, Koganei, Tokyo 184-8584, Japan (e-mail: j.yma@k.hosei.ac.jp).

Digital Object Identifier 10.1109/JLT.2008.919424

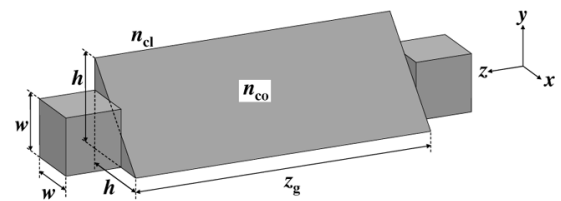

(a)

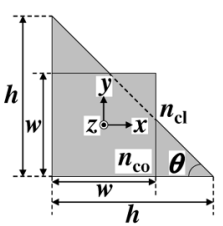

(b)

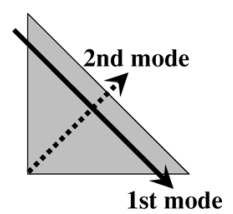

(c)
Fig. 1. Configuration of a triangular waveguide. (a) Perspective view. (b) Cross sectional view. (c) Optical axes of the two fundamental modes.

$1.55 \mu \mathrm{m}$. The extinction ratio is more than $20 \mathrm{~dB}$ over a wide wavelength range of 1.25 to $1.65 \mu \mathrm{m}$, which is not obtainable in the previous structures with a long device length. To verify the numerical results, we measured the polarization conversion behavior using a geometrically expanded model at a microwave frequency of $15 \mathrm{GHz}$.

To alleviate the fabrication difficulty, we also deal with a modified structure in which the two corners of the triangular waveguide are cut. Although the conversion length is somewhat increased, the polarization is satisfactorily converted with an insertion loss of about $0.4 \mathrm{~dB}$.

\section{BASIC STUDY}

It is well-known that an asymmetric waveguide structure allows the generation of two fundamental polarization modes that are sufficiently hybrid. To possess the polarization conversion behavior, the asymmetric waveguide must have two modes with almost the same intensity distributions, and their optical axes are perpendicular to each other. A large difference in the propagation constants is absolutely necessary to realize a short device length. This requires that the field in the optical axis of the first mode should "see" a larger core than the field of the second mode. These facts encourage us to employ the triangular waveguide shown in Fig. 1. The cross section shown in Fig. 1(b) exhibits an isosceles triangle whose side length is designated as $h$. The two congruent angles are taken to be $\theta=45^{\circ}$. In this case, the first mode has the optical axis denoted by the solid line in Fig. 1(c), and the second mode has the axis denoted by the dotted line.

The length of the triangular waveguide is designated as $z_{\mathrm{g}}$. The width of an input (or output) square waveguide is designated as $w$. The ratio of $w$ to $h$ will be determined in such a way 
that the two eigenmode fields match well with subsequent low transition loss at the junction.

The cladding refractive index $n_{\mathrm{cl}}$ is chosen to be 1.444 $\left(\mathrm{SiO}_{2}\right)$, except for experimental demonstration at a microwave frequency in Section IV, where the cladding material is taken to be air for convenience. The relative refractive index difference $\Delta n=\left(n_{\mathrm{co}}^{2}-n_{\mathrm{cl}}^{2}\right) /\left(2 n_{\mathrm{co}}^{2}\right)$ is taken to be over a range of $24 \%$ to $42 \%$. This corresponds to the core refractive index over a range of 2.0 to 3.6. This means that we include the case where the core material is chosen to be silicon nitride $(\mathrm{SiN})$ and silicon (Si) in a lightwave model.

It should be noted that the waveguide is usually fabricated on a silicon substrate. The existence of the silicon substrate may cause leakage loss becuase of its high refractive index. In this paper, we assume that the core is made far from the substrate with subsequent negligible leakage loss.

\section{A. Eigenmode Analysis}

The propagation constants are calculated using the eigenmode analysis based on the imaginary-distance YM-BPM [13]. The numerical parameters are as follows: the transverse sampling widths are $\Delta x=\Delta y=0.01 \mu \mathrm{m}$ and the longitudinal sampling width is $\Delta \tau=0.002 \mu \mathrm{m}$ (in the imaginary-distance procedure, the longitudinal axis $z$ is changed to the imaginary axis $j \tau)$. For the analysis of the waveguide with a lower core index, the sampling widths are doubled, since sufficient accuracy is obtained. The technique for averaging the permittivity is employed in the sloped side. To reduce the spurious reflection at the computational boundary, we can adopt various absorbing boundary conditions. Note that in the eigenmode analysis by the imaginary-distance BPM, the field near the computational boundary is dominated by an evanescent wave. In this analysis, we, therefore, modify the well-known transparent boundary condition [14], i.e., the field is assumed to decay exponentially without phase progression. The modified transparent boundary condition provides reasonable results with small computer memories.

We first investigate a polarization conversion length at a wavelength of $\lambda_{1.55}=1.55 \mu \mathrm{m}$. The conversion length is calculated by $L_{\mathrm{c}}=\pi /\left(\beta_{1}-\beta_{2}\right)$, where $\beta_{1}$ and $\beta_{2}$ are the propagation constants of the first and second modes of the triangular waveguide, respectively.

Fig. 2 shows the conversion length $L_{\mathrm{c}}$ as a joint function of $\Delta n$ and $h / \lambda_{1.55}$. Note that $h$ and $L_{\mathrm{c}}$ are normalized to a free-space wavelength of $\lambda_{1.55}=1.55 \mu \mathrm{m}$. If the data are normalized to the guided wavelength $\lambda_{\mathrm{g}}$, the resultant chart can be used in any wavelength. However, since $\lambda_{\mathrm{g}}$ becomes a function of $h$, it is difficult to draw the chart normalized to $\lambda_{\mathrm{g}}$. Therefore, we plot the data normalized to the free-space wavelength. This means that Fig. 2 can be approximately used in other wavelengths. The difference in the polarization conversion lengths when $h$ is normalized to other wavelengths will be found in Fig. 13.

The conversion length tends to decrease as the relative refractive index difference is increased. For example, a conversion length of $1.9 \mu \mathrm{m}\left(L_{\mathrm{c}} / \lambda_{1.55} \simeq 1.23\right)$ is obtained for $n_{\mathrm{co}} \simeq$ $3.5(\Delta n \simeq 41.5 \%)$ and $h \simeq 0.4 \mu \mathrm{m}\left(h / \lambda_{1.55} \simeq 0.26\right)$. Fig. 2 also suggests that an appropriate combination of $h$ and $\Delta n$ leads

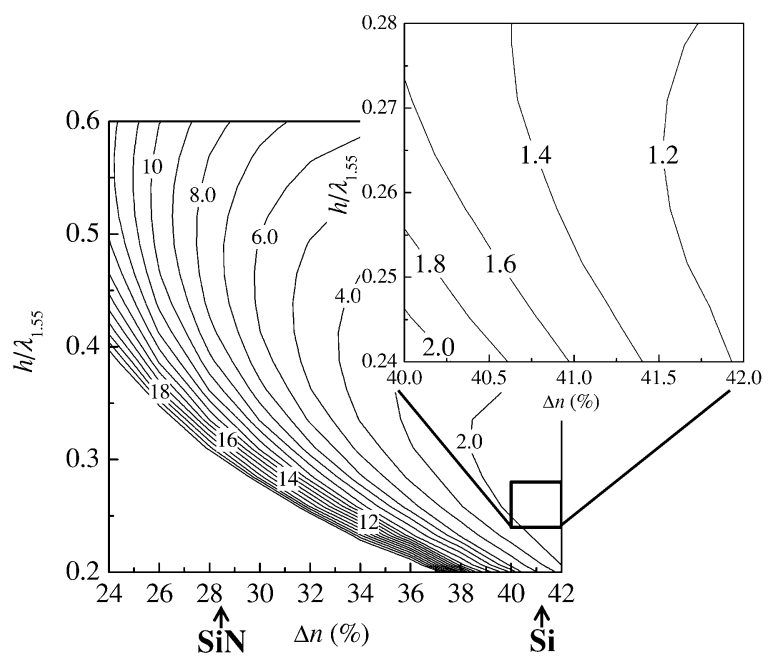

Fig. 2. Normalized polarization conversion length $L_{\mathrm{c}} / \lambda_{1.55}\left(n_{\mathrm{cl}}=1.444\right)$.

to the wideband operation of the polarization conversion. Note that the variation of $h$ corresponds to the variation of wavelength. Therefore, when we choose the geometrical parameters in the region where the contour line $L_{\mathrm{c}}$ is nearly vertical, $L_{\mathrm{c}}$ is insensitive to the change in wavelength. Furthermore, a short device length generally contributes to the wideband operation of the conversion behavior. The detailed discussion will be made later in Fig. 11. On the basis of the aforementioned results, the $\equiv$ ctive indices of the core and the triangular waveguides are, respectively, fixed to be $n_{\mathrm{co}}=3.476(\mathrm{Si})(\Delta n=41.37 \%)$ and $h=0.42 \mu \mathrm{m}\left(h / \lambda_{1.55} \simeq 0.271\right)$ unless otherwise noted.

Figs. 3 and 4 show the eigenmode field distributions. Fig. 3 shows the first mode fields obtained from the imaginary-distance YM-BPM. The second mode fields shown in Fig. 4 can be generated by subtracting the obtained first mode fields using the Gram-Schmidt orthogonalization technique [13]. In this case, the propagation constants are calculated to be $\beta_{1}=8.836 \mathrm{rad} / \mu \mathrm{m}$ and $\beta_{2}=7.190 \mathrm{rad} / \mu \mathrm{m}$.

Using the obtained fields, we next consider the insertion loss as a function of input waveguide width to find an appropriate ratio of $w / h$. Mode-field matching is absolutely necessary to reduce the transition loss between the input and triangular waveguides. The field matching also contributes to reduction in the reflection loss [7]. Taking into account the center of gravity of the field distributions between the input and triangular waveguides, it is expected that the input waveguide width should be smaller than the side length of the triangular waveguide, i.e., $w / h<1$.

The transition loss may be evaluated from the overlap integral of the eigenmode fields of the input and triangular waveguides. For the full vector fields, the overlap integral may be evaluated by [15], [16]

$$
\eta=\sqrt{\eta_{\mathrm{a}} \eta_{\mathrm{b}}}
$$

where

$$
\begin{aligned}
\eta_{\mathrm{a}} & =\frac{\left|\iint \mathbf{E}_{\mathrm{in}} \times \mathbf{H}_{\mathrm{tr}} \cdot \mathbf{n}_{z} d S\right|^{2}}{\operatorname{Re}\left\{\iint \mathbf{E}_{\mathrm{in}} \times \mathbf{H}_{\mathrm{in}}^{*} \cdot \mathbf{n}_{z} d S\right\} \operatorname{Re}\left\{\iint \mathbf{E}_{\mathrm{tr}} \times \mathbf{H}_{\mathrm{tr}}^{*} \cdot \mathbf{n}_{z} d S\right\}} \\
\eta_{\mathrm{b}} & =\frac{\left|\iint \mathbf{E}_{\mathrm{tr}} \times \mathbf{H}_{\mathrm{in}} \cdot \mathbf{n}_{z} d S\right|^{2}}{\operatorname{Re}\left\{\iint \mathbf{E}_{\mathrm{in}} \times \mathbf{H}_{\mathrm{in}}^{*} \cdot \mathbf{n}_{z} d S\right\} \operatorname{Re}\left\{\iint \mathbf{E}_{\mathrm{tr}} \times \mathbf{H}_{\mathrm{tr}}^{*} \cdot \mathbf{n}_{z} d S\right\}}
\end{aligned}
$$



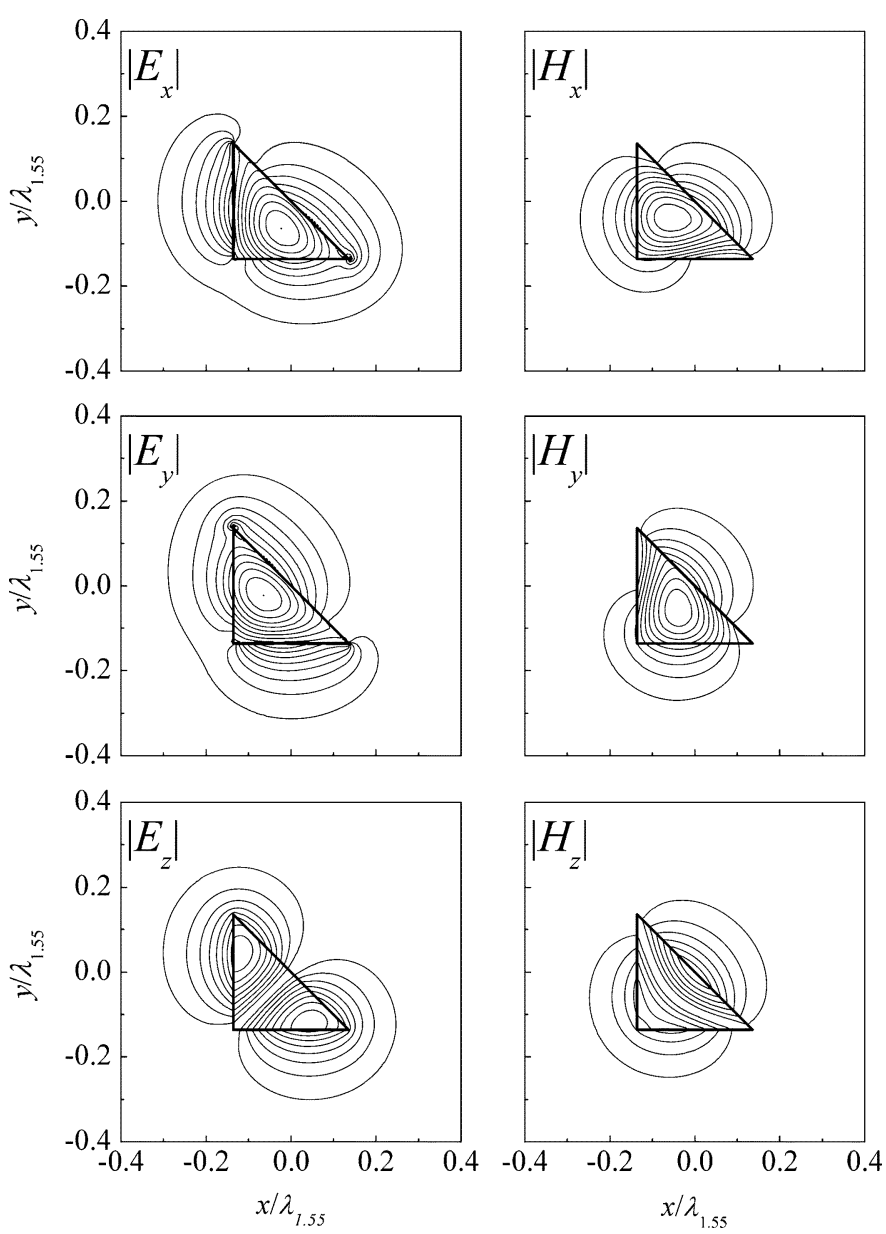

Fig. 3. Eigenmode fields of the first mode.

in which $\left(\mathbf{E}_{\mathrm{in}}, \mathbf{H}_{\mathrm{in}}\right)$ and $\left(\mathbf{E}_{\mathrm{tr}}, \mathbf{H}_{\mathrm{tr}}\right)$ are, respectively, the eigenmode fields of the input and triangular waveguides. The integration is carried out over the junction plane. Since the two orthogonal modes (the first and second modes) are excited, we separately calculate $\eta_{1}$ and $\eta_{2}$, and add these values to estimate the total transition loss.

Note that (1) yields an approximate value, since the evanescent wave caused in the propagation direction cannot be taken into account. Fortunately, Fresnel reflection loss generated from the difference in the phase constants of the input and triangular waveguides is sufficiently small. As a result, the insertion loss of the triangular waveguide can be estimated by twice the transition loss, since there exist the two junction planes.

Fig. 5 shows the estimated insertion loss against $w / h$. It is confirmed that a minimum insertion loss of $0.3 \mathrm{~dB}$ is obtained with $w / h \simeq 0.65$, which means $w=0.27 \mu \mathrm{m}\left(w / \lambda_{1.55} \simeq\right.$ $0.174)$ for $h=0.42 \mu \mathrm{m}\left(h / \lambda_{1.55} \simeq 0.271\right)$. For comparison, the results for $n_{\mathrm{co}}=2.2(\mathrm{SiN})(\Delta n=28.46 \%)$, in which $h$ is $0.7 \mu \mathrm{m}\left(h / \lambda_{1.55} \simeq 0.45\right)$, are also presented. It is seen that an optimum ratio is not sensitive to the change in $\Delta n$ (mainly determined by geometry), and that the insertion loss reduces as $\Delta n$ is decreased, although the conversion length is increased, as found in Fig. 2.

Fig. 6 shows the insertion loss and the conversion length as a function of $\Delta n$. The configuration parameters $h / \lambda_{1.55}$ and $w / \lambda_{1.55}$ are chosen to be optimal values, in which both loss and
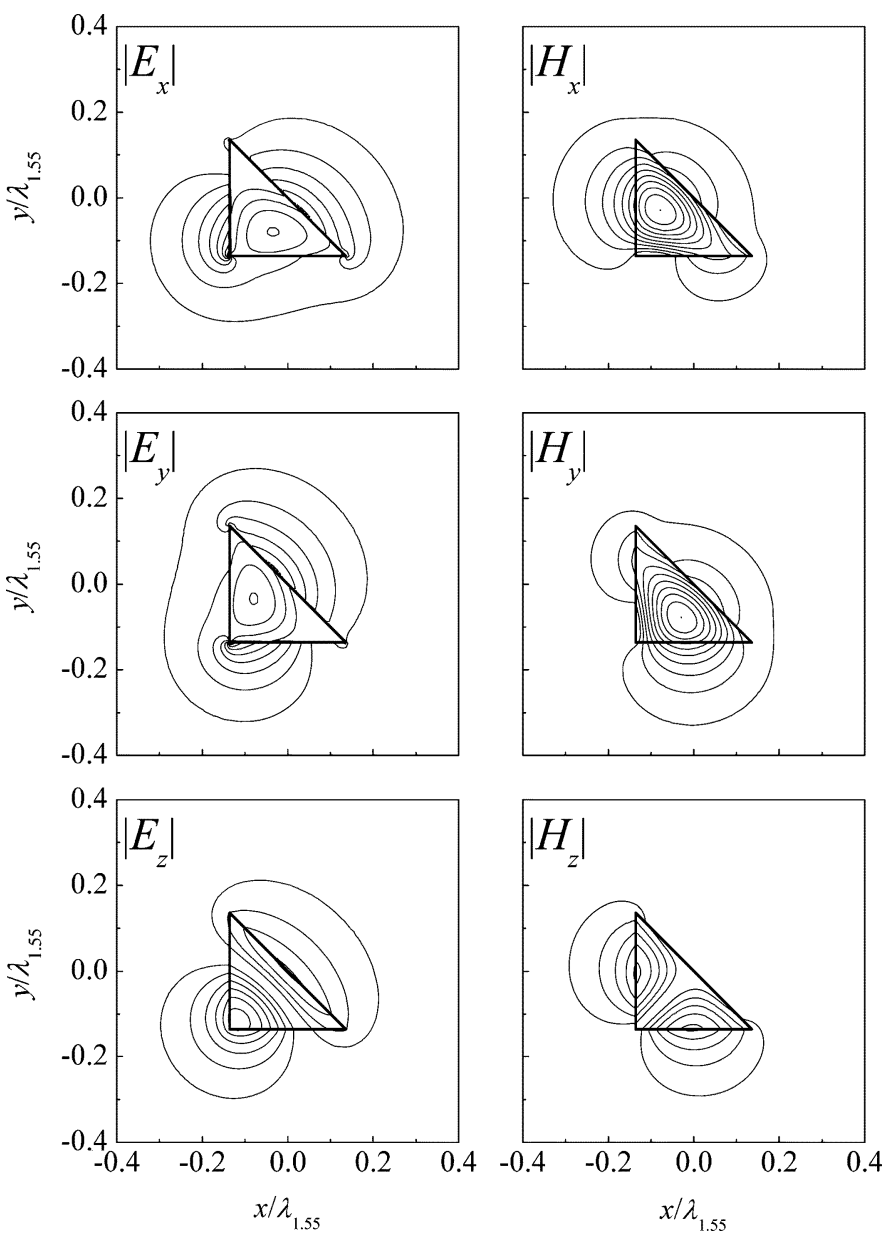

Fig. 4. Eigenmode fields of the second mode.

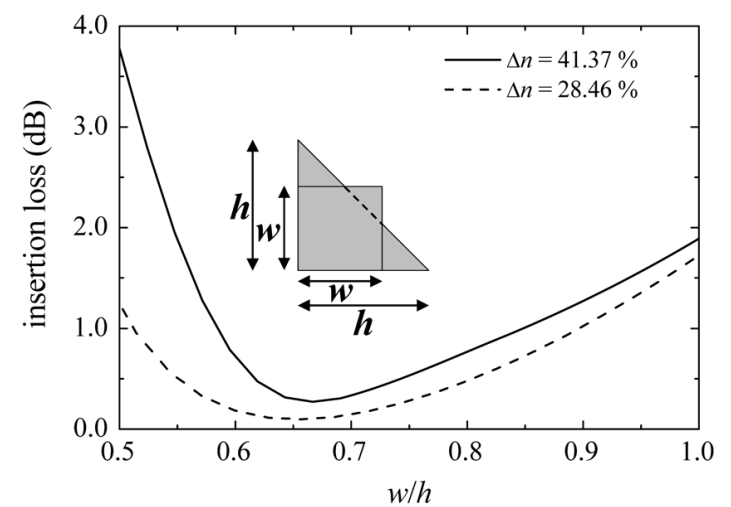

Fig. 5. Insertion loss evaluated from the eigenmode analysis as a function of $w / h$.

conversion length are minimized. It is seen that the conversion length is about $2 \mu \mathrm{m}\left(L_{\mathrm{c}} / \lambda_{1.55}=1.23\right)$ for $n_{\mathrm{co}}=3.476(\Delta n=$ $41.37 \%)$ and the loss is about $0.3 \mathrm{~dB}$. For $n_{\mathrm{co}}=2.2(\Delta n=$ $28.46 \%$ ), the loss is reduced to less than $0.1 \mathrm{~dB}$, although the conversion length is increased to about $11 \mu \mathrm{m}\left(L_{\mathrm{c}} / \lambda_{1.55}=\right.$ 7.1).

\section{B. Propagating Beam Analysis}

We next carry out the propagating beam analysis using the FDTD method. The transverse sampling widths are the same as 


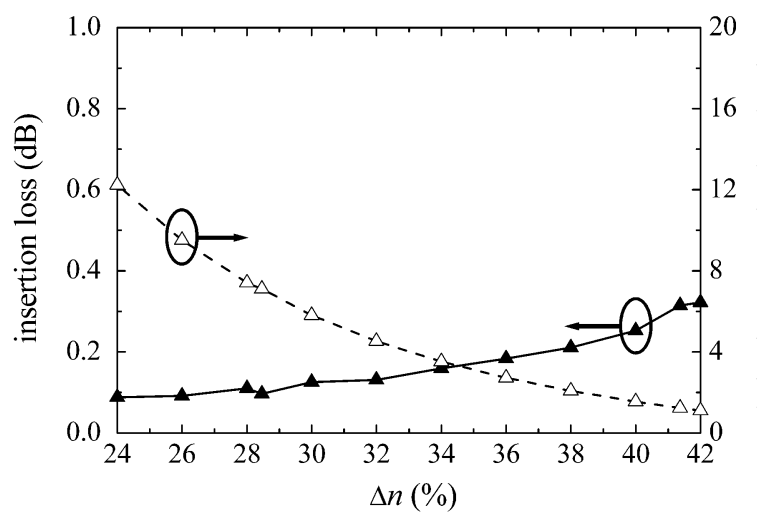

Fig. 6. Insertion loss and polarization conversion length $L_{c} / \lambda_{1.55}$ evaluated from the eigenmode analysis as a function of $\Delta n\left(n_{\mathrm{cl}}=1.444\right)$.
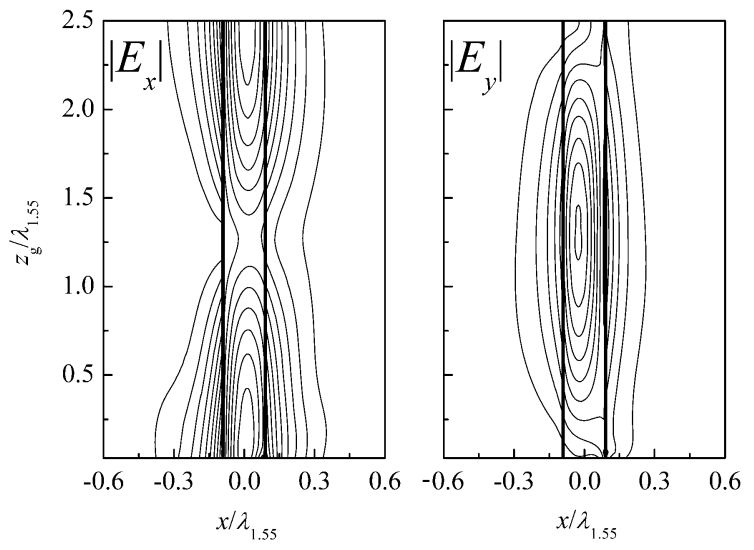

Fig. 7. Field distributions in the $x-z$ plane.

those used in the eigenmode analysis. The longitudinal sampling width is taken to be $\Delta z=0.005 \mu \mathrm{m}$. The configuration parameters are the same as those of the silicon core model in the previous section, i.e., $n_{\text {co }}=3.476(\Delta n=41.37 \%), h=0.42 \mu \mathrm{m}$ $\left(h / \lambda_{1.55} \simeq 0.271\right)$, and $w=0.27 \mu \mathrm{m}\left(w / \lambda_{1.55} \simeq 0.174\right)$. The electric field distributions in the $x-z$ plane are illustrated in Fig. 7. It is observed that the incident $E_{11}^{x}$ mode is converted into the $E_{11}^{y}$ mode at $z_{\mathrm{g}} / \lambda_{1.55}=1.23$.

Fig. 8 shows the guided-mode power against the triangular waveguide length. It is again seen that almost complete polarization conversion can be obtained at $z_{\mathrm{g}} / \lambda_{1.55}=1.23$. This result is in good agreement with the eigenmode analysis. Since the insertion loss is evaluated to be about $0.5 \mathrm{~dB}$ with negligible reflection loss, the insertion loss obtained from the propagating beam analysis fairly agrees with the loss estimated in Fig. 5 .

To know fabrication tolerance of the interior angle, we evaluate the extinction ratio as a function of interior angle $\theta$ shown in the inset of Fig. 9. Note that the side length $h^{\prime}$ varies with $h$ being fixed, when the interior angle is changed. The data are presented for the incident waves of not only the $E_{11}^{x}$ mode but also the $E_{11}^{y}$ mode. It is seen that interior angles of $45 \pm 1^{\circ}$ are required to maintain an extinction ratio of more than $20 \mathrm{~dB}$.

\section{WAVELENGTH ChaRACTERISTICS}

We now investigate the polarization conversion property as a function of wavelength. The core and cladding refractive indices are assumed to vary with the Sellmeier-type dispersion formula

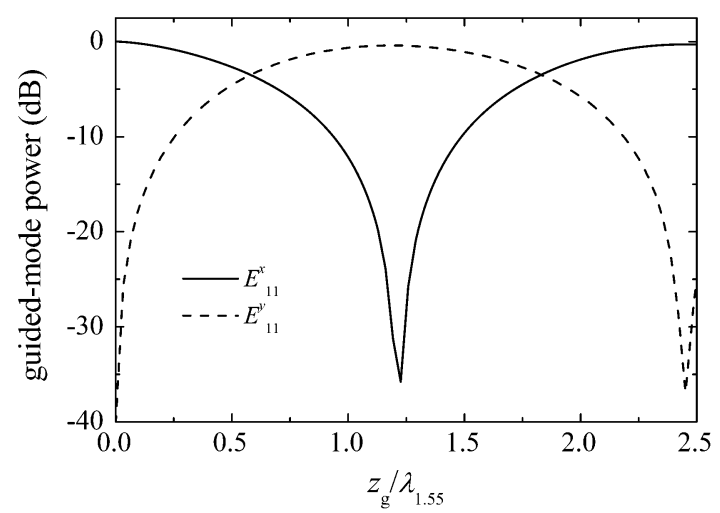

Fig. 8. Guided-mode power as a function of $z_{g} / \lambda_{1.55}$.

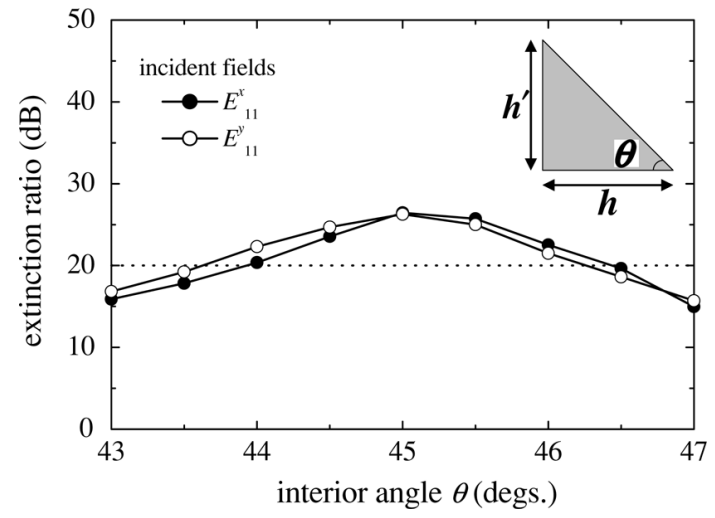

Fig. 9. Extinction ratio as a function of interior angle $\theta\left(z_{\mathrm{g}} / \lambda_{1.55} \simeq 1.26\right)$.

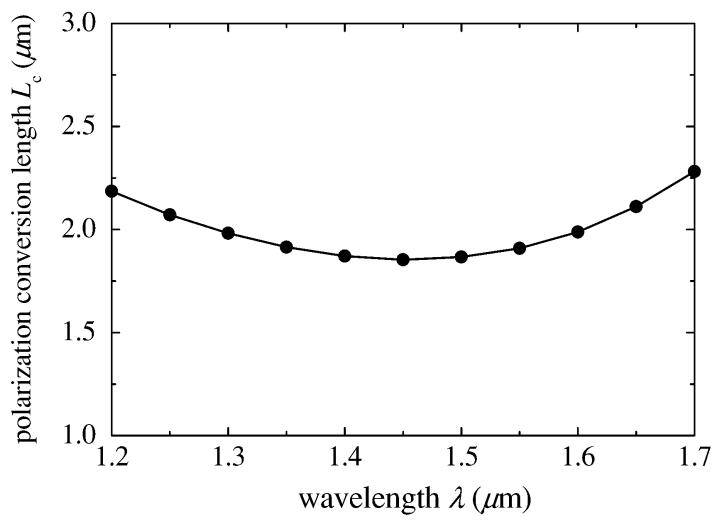

Fig. 10. Polarization conversion length $L_{\mathrm{c}}$ evaluated from the eigenmode analysis as a function of wavelength.

[17]. Fig. 10 shows the wavelength response of the polarization conversion length. It is seen that the conversion length is almost $2 \mu \mathrm{m}$ over a wavelength range of 1.25 to $1.65 \mu \mathrm{m}$. Since the conversion length is minimal at $\lambda=1.45 \mu \mathrm{m}$, the wideband operation around $\lambda=1.45 \mu \mathrm{m}$ is expected.

Fig. 11 shows the extinction ratio as a function of wavelength. As expected, an extinction ratio of more than $20 \mathrm{~dB}$ is obtained over a wide wavelength range of 1.25 to $1.65 \mu \mathrm{m}$ for $z_{\mathrm{g}}=1.95 \mu \mathrm{m}\left(z_{\mathrm{g}} / \lambda_{1.55} \simeq 1.26\right)$. The insertion loss is also shown in Fig. 11, where the loss is calculated by the propagating beam analysis. It is seen that the loss is less than $0.5 \mathrm{~dB}$ over a 


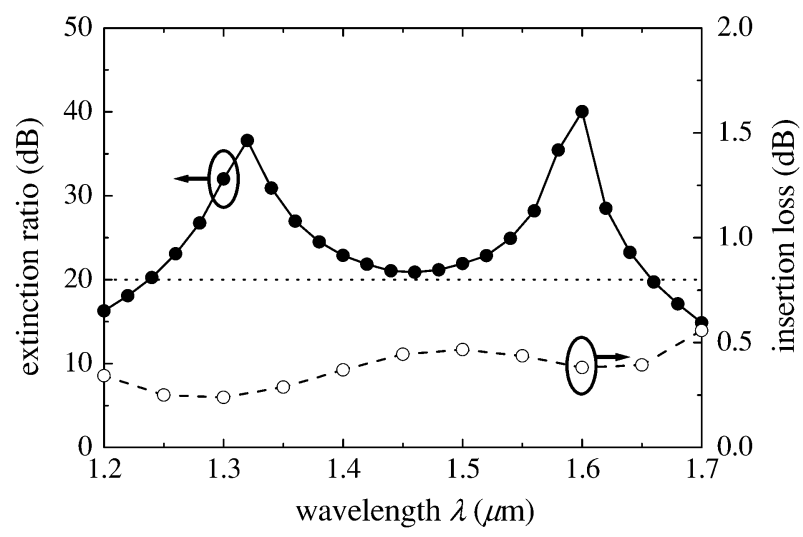

Fig. 11. Extinction ratio and insertion loss as a function of wavelength $\left(z_{\mathrm{g}} / \lambda_{1.55} \simeq 1.26\right)$

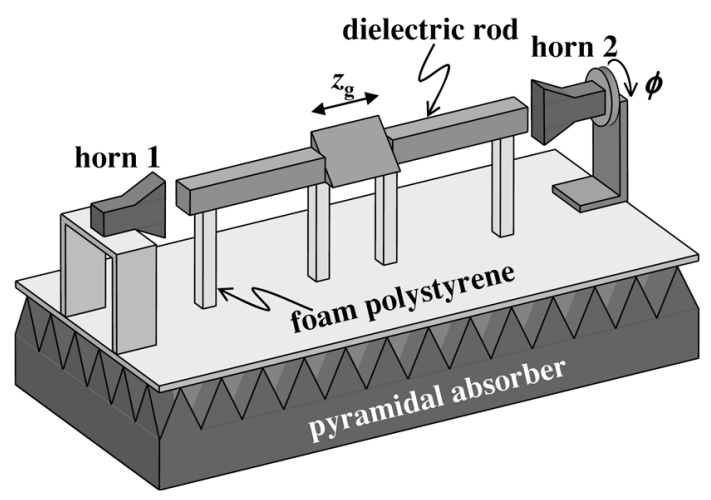

Fig. 12. Experimental setup.

wavelength range of 1.2 to $1.7 \mu \mathrm{m}$. The slight oscillatory behavior is mainly due to the coherent coupling between the radiation fields generated at the two junctions [18], which cannot be taken into account in the modal analysis in Section II-A.

\section{EXPERIMENT}

So far, we have numerically investigated the polarization conversion characteristics of a triangular waveguide at lightwave frequencies. To validate the numerical results, we next experimentally examine the characteristics at a microwave frequency. The use of microwave leads to geometrical expansion with subsequent decrease in the sensitivities to fabrication and measurement [19].

A microwave frequency of $f=15 \mathrm{GHz}(\lambda=20 \mathrm{~mm})$ is adopted. The waveguide is made of a polycarbonate whose relative permittivity is $\epsilon_{\mathrm{r}}=2.8$. Since the cladding material is taken to be air, $\Delta n$ becomes $32.14 \%$. The dimension of the cross section of the square waveguide connected to the triangular waveguide is $w=7.5 \mathrm{~mm}(w / \lambda=0.375)$. The side length of the triangular waveguide is $h=12 \mathrm{~mm}(h / \lambda=0.6)$, so that $w / h=0.625$. The polarization conversion in the triangular waveguide is measured using the experimental setup illustrated in Fig. 12. The triangular and square waveguides are supported by form-polystyrene pillars and the input square waveguide is fed by a horn launcher 1 through a metallic waveguide (WR-51). The dimensions of the horn aperture are $49 \mathrm{~mm} \times 66 \mathrm{~mm}$. When the metallic waveguide is excited with the fundamental mode whose major electric field component is $E_{x}$, most of the power

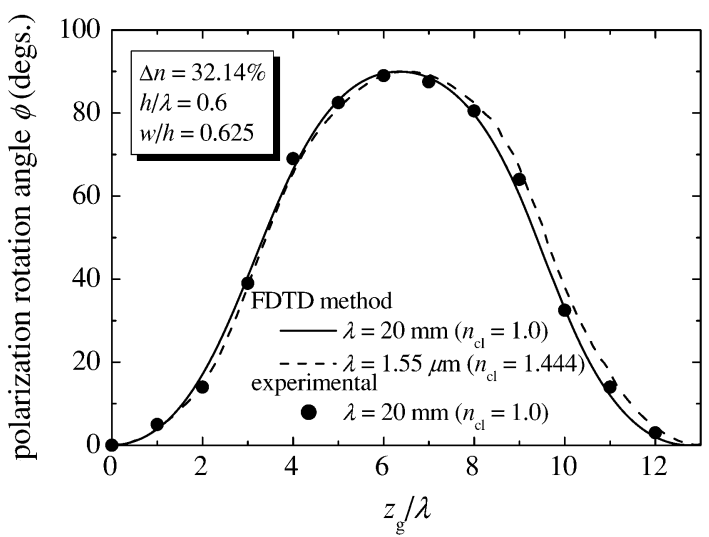

Fig. 13. Polarization rotation angle $\phi$ as a function of $z_{\mathrm{g}} / \lambda$.

is converted into the $E_{11}^{x}$ mode power. The polarization rotation angle $\phi$ is measured using the receiving horn 2 .

Fig. 13 shows the comparison of polarization rotation angles between the experimental and numerical results. Note that the two calculated results are provided: one is for a microwave model, and the other for a lightwave model. Both calculated data are for $\Delta n=32.14 \%$. Although both data are slightly different because of the difference in the refractive indexes of the claddings, i.e., $n_{\mathrm{cl}}$ is taken to be unity for the microwave model, and that is 1.444 for the lightwave model, their similar property verifies the validity of confirming the operation using the microwave model. (Note that $z_{\mathrm{g}}$ is normalized to free-space wavelength $\lambda$ in Fig. 13. Both results become identical when $z_{\mathrm{g}}$ is normalized to the guided wavelength $\lambda_{\mathrm{g}}$.) In this model, almost complete polarization conversion is observed at $z_{\mathrm{g}} / \lambda=6.5$. A good correlation is found to exist between the experimental and numerical results. Further experiment demonstrates that the insertion loss of the triangular waveguide is nearly negligible (less than $0.3 \mathrm{~dB}$ ), which again shows good agreement with the numerical results.

\section{Modified Structure}

In the preceding sections, we have studied the polarization conversion characteristics of the triangular waveguide. The triangular configuration is ideal to achieve an extremely short device length. It is, however, not easy to fabricate the triangular waveguide at a lightwave frequency. In this section, we, therefore, investigate a more practical configuration, although the conversion length is somewhat increased.

A more practical configuration is shown in Fig. 14, in which the two corners of the triangular waveguide are cut and the cut plane is aligned with the square waveguide. In other words, one of the corners of the square waveguide is cut with an angle of $45^{\circ}$. Preliminary calculation shows that when the two corners of the triangular waveguide are cut, while maintaining the same configuration parameters as those in Section II, the conversion length is increased to $5.3 \mu \mathrm{m}\left(L_{\mathrm{c}} / \lambda_{1.55} \simeq 3.42\right)$. We, therefore, slightly increase the width $w$. After some auxiliary calculations, we choose $w=0.32 \mu \mathrm{m}\left(w / \lambda_{1.55} \simeq 0.21\right), \gamma=0.13 \mu \mathrm{m}$ $\left(\gamma / \lambda_{1.55} \simeq 0.08\right)$, and $z_{\mathrm{g}}=2.75 \mu \mathrm{m}\left(z_{\mathrm{g}} / \lambda_{1.55} \simeq 1.77\right)$.

Fig. 15 shows the extinction ratio as a function wavelength. The data obtained with the original (triangular) structure are 


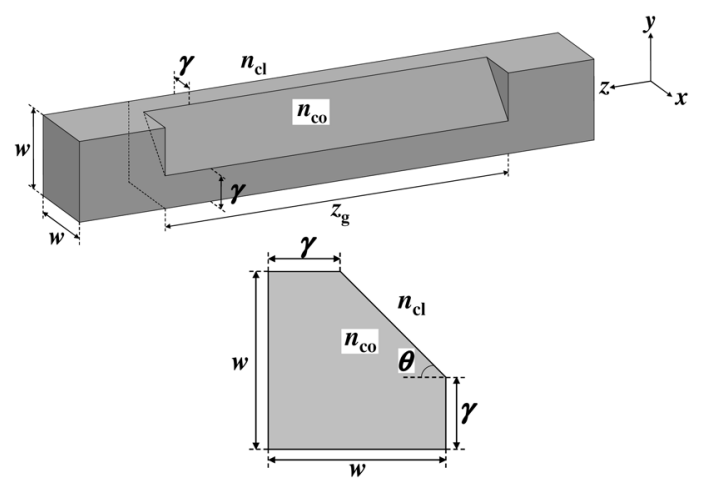

Fig. 14. Configuration of a modified structure.

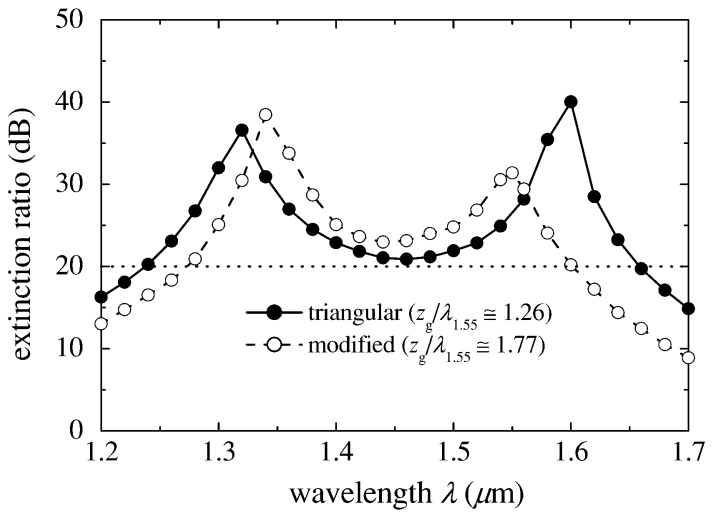

Fig. 15. Extinction ratio as a function of wavelength.

again plotted for reference. It is found that the wideband operation comparable to that with the original structure is maintained, although the device length is somewhat lengthened. An extinction ratio of more than $20 \mathrm{~dB}$ is obtained over a wavelength range of 1.3 to $1.6 \mu \mathrm{m}$. The insertion loss is evaluated to be about $0.4 \mathrm{~dB}$ over the same wavelength range.

\section{CONCLUSION}

An extremely short polarization converter using a triangular waveguide has been proposed and investigated. The polarization conversion length against the core refractive index and the side length of the triangular waveguide is calculated by the eigenmode analysis using the imaginary-distance beam-propagation method based on Yee's mesh. It is revealed at a wavelength of $1.55 \mu \mathrm{m}$ that the conversion length decreases from 11 to $2 \mu \mathrm{m}$, as the core refractive index is increased from 2.2 to 3.476 for a silica cladding. The minimum insertion loss is obtained for $w / h \simeq 0.65$, where $w$ is the width of the input (or output) waveguide and $h$ is the side length of the triangular waveguide.

The polarization conversion behavior has been analyzed by the FDTD method. It is found for a silicon core with a silica cladding that the conversion length is $2 \mu \mathrm{m}$ and the insertion loss is about $0.5 \mathrm{~dB}$ at a wavelength of $1.55 \mu \mathrm{m}$. An extinction ratio of more than $20 \mathrm{~dB}$ is obtained over a wavelength range of 1.25 to $1.65 \mu \mathrm{m}$. The polarization conversion behavior was confirmed by the experiment using a geometrically expanded model at a microwave frequency of $15 \mathrm{GHz}$.

Further consideration has been devoted to a more practical model in which only the one corner of the square waveguide is cut with an angle of $45^{\circ}$. An extinction ratio of more than $20 \mathrm{~dB}$ is obtained over a wavelength range of 1.3 to $1.6 \mu \mathrm{m}$ with a device length of about $2.8 \mu \mathrm{m}$.

\section{ACKNOWLEDGMENT}

The authors would like to thank Prof. Toshihiko Baba of Yokohama National University for suggesting the study of the modified structure in Section V.

\section{REFERENCES}

[1] T. Barwicz, M. R. Watts, M. A. Popović, P. T. Rakich, L. Socci, F. X. Kärtner, E. P. Ippen, and H. I. Smith, "Polarization-transparent microphotonic devices in the strong confinement limit," Nature Photon., vol. 1, no. 1, pp. 57-60, Jan. 2007.

[2] Y. Shani, R. Alferness, T. Koch, U. Koren, M. Oron, B. I. Miller, and M. G. Young, "Polarization rotation in asymmetric periodic loaded rib waveguides," Apply. Phys. Lett., vol. 59, no. 11, pp. 1278-1280, Sep. 1991.

[3] V. P. Tzolov and M. Fontaine, "A passive polarization converter free of longitudinally-periodic structure," Opt. Commun., vol. 127, pp. 7-13, Jun. 1996.

[4] J. Z. Huang, R. Scarmozzino, G. Nagy, M. J. Steel, and R. M. Osgood Jr., "Realization of a compact and single-mode optical passive polarization converter," IEEE Photon. Technol. Lett., vol. 12, no. 3, pp. 317-319, Mar. 2000.

[5] B. M. A. Rahman, S. S. A. Obayya, N. Somasiri, M. Rajarajan, K. T. V. Grattan, and H. A. El-Mikathi, "Design and characterization of compact single-section passive polarization rotator," J. Lightw. Technol., vol. 19, no. 4, pp. 512-519, Apr. 2001.

[6] D. Correia, J. P. da Silva, and H. E. Hernández-Figueroa, "Genetic algorithm and finite-element design of short single-section passive polarization converter," IEEE Photon. Technol. Lett., vol. 15, no. 7, pp. 915-917, Jul. 2003.

[7] J. Yamauchi, I. Ohba, T. Ando, and H. Nakano, "FDTD analysis of a polarization conversion waveguide with a single sloped sidewall," in Integ. Photon. Res., Washington, DC, Jun. 2003, pp. 198-200.

[8] H. Deng, D. O. Yevick, C. Brooks, and P. E. Jessop, "Design rules for slanted-angle polarization rotators," J. Lightw. Technol., vol. 23, no. 1, pp. 432-445, Jan. 2005.

[9] L. M. Augustin, J. J. G. M. van der Tol, E. J. Geluk, and M. K. Smit, "Short polarization converter optimized for active-passive integration in InGaAsP-InP," IEEE Photon. Technol. Lett., vol. 19, no. 20, pp. 1673-1675, Oct. 2007.

[10] M. R. Watts and H. A. Haus, "Integrated mode-evolution-based polarization rotators," Opt. Lett., vol. 30, no. 2, pp. 138-140, Jan. 2005.

[11] H. Fukuda, K. Yamada, T. Tsuchizawa, T. Watanabe, and S. Itabashi, "Ultrasmall and wideband polarization rotator based on silicon wire waveguides," in Integ. Photon. Nanophoton. Res. Appl., Salt Lake City, UT, Jul. 2007.

[12] J. Yamauchi, M. Yamanoue, and H. Nakano, "An extremely short polarization converter using a triangular waveguide," in Integ. Photon. Nanophoton. Res. Appl., Salt Lake City, UT, Jul. 2007.

[13] S. M. Lee, "Finite-difference vectorial-beam-propagation method using Yee's discretization scheme for modal fields,"J. Opt. Soc. Amer. A, vol. 13, no. 7, pp. 1369-1377, Jul. 1996.

[14] G. R. Hadley, "Transparent boundary condition for beam propagation," Opt. Lett., vol. 16, no. 9, pp. 624-626, May 1991.

[15] D. Marcuse, "Excitation of the dominant mode of a round fiber by a Gaussian beam," Bell Syst. Tech. J., vol. 49, no. 8, pp. 1695-1703, Oct. 1970.

[16] C. Vassallo, "On a rigorous calculation of the efficiency for coupling light power into optical waveguides," IEEE J. Quantum Electron., vol. QE-13, no. 4, pp. 165-173, Apr. 1977.

[17] E. D. Palik, Ed., Handbook of Optical Constants of Solids. San Diego, CA: Academic, 1985, pp. 547-569.

[18] L. M. Johnson and D. Yap, "Theoretical analysis of coherently coupled optical waveguide bends," Appl. Opt., vol. 23, no. 17, pp. 2988-2990, Sep. 1984.

[19] T. Ando, T. Murata, H. Nakayama, J. Yamauchi, and H. Nakano, "Analysis and measurement of polarization conversion in a periodically loaded dielectric waveguide," IEEE Photon. Technol. Lett., vol. 14, no. 9, pp. 1288-1290, Sept. 2002. 


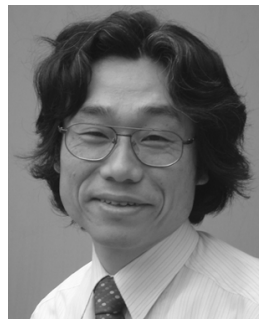

Junji Yamauchi (M'84) was born in Nagoya, Japan, on August 23, 1953. He received the B.E., M.E., and Dr.Eng. degrees from Hosei University, Tokyo, Japan, in 1976, 1978, and 1982, respectively.

From 1984 to 1988 , he served as a Lecturer in the Electrical Engineering Department, Tokyo Metropolitan Technical College. Since 1988, he has been a member of the faculty of Hosei University, Tokyo, Japan, where he is now a Professor of electronic informatics. His research interests include optical waveguides and circularly polarized antennas. He is the author of Propagating Beam Analysis of Optical Waveguides (Research Studies Press, 2003).

Dr. Yamauchi is a member of the Optical Society of America and the Institute of Electronics, Information, and Communication Engineers of Japan.

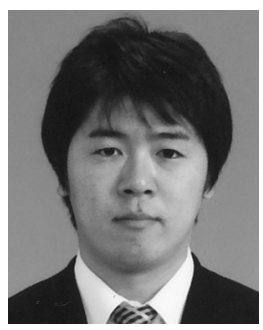

Masahiro Yamanoue was born in Yokohama, Japan, on October 18, 1983. He received the B.E. degree from Hosei University, Tokyo, Japan, in 2002, where he is currently working toward the M.E. degree.

Mr. Yamanoue is a Student Member of the Institute of Electronics, Information and Communication Engineers of Japan.

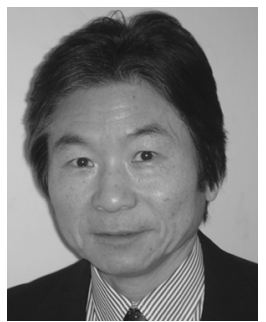

Hisamatsu Nakano (M'75-SM'87-F'92) was born in Ibaraki, Japan, on April 13, 1945. He received the B.E., M.E., and Dr.Eng. degrees in electrical engineering from Hosei University, Tokyo, Japan, in 1968, 1970, and 1974, respectively.

Since 1973, he has been a member of the faculty of Hosei University, where he is now a Professor of electronic informatics. His research topics include numerical methods for low- and high-frequency antennas and optical waveguides. He has published more than 200 refereed journal papers, more than 200 international symposium papers, and more than 750 national symposium papers. He is the author of a book entitled Helical and Spiral Antennas (Wiley, 1987) and the coauthor of Analysis Methods of Electromagnetic Wave Problems, Volume Two (Artech House, 1986). In addition, he is the author of Helical and Spiral Antennas, Encyclopedia of Communications (Wiley, 2002).

Dr. Nakano received the IEE International Conference on Antennas and Propagation Best Paper Award and the IEEE TRANSACTIONS ON ANTENNAS AND Propagation Best Application Paper Award (H. A. Wheeler Award) in 1989 and 1994, respectively. In 1992, he was elected an IEEE Fellow for contributions to the design of spiral and helical antennas. In 2001, he received the Award of Distinguished Technical Communication (from the Society for Technical Communication, USA) and the Science and Technology Progress Award (from Hangzhou, China). He was also the recipient of the Chen-To Tai Distinguished Educator Award (from the IEEE Antennas and Propagation Society) in 2006. $\mathrm{He}$ is an Associate Editor of several journals and magazines, such as Electromagnetics, IEEE ANTENNAS AND PROPAGATION MAGAZINE, IEEE ANTENNAS AND WirELESS PROPAGATION LETTERS, and Asian Information-Science-Life. 